\title{
Aider les élèves en difficulté à réussir
}

Dernière mise à jour : 2 mars 2017



Plus d'un quart des élèves de 15 ans des pays de l'OCDE n'atteint pas le niveau de compétence de base en mathématiques, compréhension de l'écrit et sciences. Dans d'autres pays, cette proportion est souvent bien plus importante. Ces piètres résultats scolaires ont de graves conséquences pour les individus : les élèves faibles sont en général moins motivés et manquent de confiance en eux, et sont exposés au risque d'absentéisme. À long terme, cela les pénalise et compromet l'avenir économique et social du pays.

Une publication de l'OCDE sur les élèves en difficulté (Low-Performing Students: Why they Fall Behind and How To Help Them) analyse le milieu familial, le parcours scolaire et les attitudes à l'égard de l'école. Un élève est faible lorsqu'il ne parvient pas à atteindre le niveau 2 à l'enquête mondiale PISA de l'OCDE réalisée auprès 
des jeunes de 15 ans. Cela signifie qu'il a généralement du mal à comprendre une notice, telle que celle figurant dans une boîte d'aspirine. Selon le rapport, la faiblesse en mathématiques est moins fréquente dans les pays où les ressources éducatives sont réparties plus équitablement entre les écoles. Bien sûr, les enseignants comptent aussi : un élève dont le professeur n'a guère d'attentes ou est souvent absent a plus de risques d'être en difficulté.

Bien que quelques pays (Italie ou Mexique) aient réussi à réduire leur proportion d'élèves faibles en mathématiques, les progrès sont globalement rares.

Il est toutefois possible d'améliorer la situation par des politiques adéquates, par exemple des programmes adaptés aux groupes vulnérables (en France, $30 \%$ des élèves en difficulté viennent de l'immigration) ou au sexe. Au Chili, $58 \%$ des élèves faibles sont des filles.

Impliquer officiellement les parents dans la gestion de l'école, dans les conseils d'établissements par exemple, a également fait ses preuves, notamment au Japon.

Neila Bachene

Voir http://www.oecd.org/pisa/aboutpisa/pisa-en-francais.htm

\section{Références}

OCDE (2016), Low-Performing Students: Why They Fall Behind and How To Help Them Succeed, PISA, Éditions OCDE http://dx.doi.org/http://dx.doi.org/ 10.1787/9789264250246-en

Aider les élèves en difficulté à réussir http://dx.doi.org/10.1787/9789264250246$\underline{\text { en }}$ 